\title{
Novel hormonal agents for metastatic Castration-Resistant Prostate Cancer: comparing outcomes. A single-center retrospective study
}

\author{
Roberto Saldanha Jarimba ${ }^{1}$, Miguel Nobre Eliseu ${ }^{1}$, João Pedroso Lima ${ }^{1}$, Vasco Quaresma ${ }^{1}$, \\ Pedro Moreira ${ }^{1}$, Pedro Coelho Nunes ${ }^{1,2}$, Edgar Tavares da Silva ${ }^{1,2}$, Arnaldo José Figueiredo ${ }^{1,2}$ \\ ${ }^{1}$ Urology and Renal Transplantation Department, Centro Hospitalar e Universitário de Coimbra, Coimbra, Portugal; \\ ${ }^{2}$ Faculty of Medicine, University of Coimbra, Portugal.
}

\begin{abstract}
Summary Introduction: Prostate cancer is the most common cancer in men, accounting for $15 \%$ of all diagnosed cancers and is the sixth leading cause of cancerrelated deaths amongst men worldwide. Abiraterone and enzalutamide were the first two novel hormonal agents approved for the treatment of metastatic prostate cancer but there is a lack of quality evidence regarding which is associated with better outcomes and who would benefit the most with one or another of these drugs.

Objective: To evaluate the clinical outcomes of real-world patients submitted to treatment with novel hormonal agents, enzalutamide and abiraterone, for castration resistant metastatic prostate cancer in an academic center.

Patients and methods: We retrospectively reviewed patients treated for castration-resistant prostate cancer with either abiraterone or enzulatamide between January 1, 2016 and December 31, 2019. The primary endpoints were biochemical response, biochemical progression, radiological progression, clinical deterioration (attributed to disease progression) and death.

Results: Enzalutamide had a higher biochemical response rate than abiraterone in patients with $\mathrm{mCRPC}(77.1 \%$ vs $58.1 \%$, $p=0.016)$. Achieving a biochemical response was associated with a lower risk of biochemical progression (OR: 0.248, $p=0.017$ ) and death (OR: 0.302, $p=0.038$ ).

Conclusions: Enzalutamide conferred higher biochemical response rate than abiraterone in patients with $\mathrm{mCRPC}$. Despite the trend to better performance of other endpoints in the enzalutamide group, it did not achieve statistical significance. Well-designed prospective studies are needed to elucidate the comparative efficacies of these agents.
\end{abstract}

KEY WORDS: Prostate cancer; Abiraterone; Enzalutamide; Castration-resistant.

Submitted 7 June 2021; Accepted 15 August 2021

\section{INTRODUCTION}

Prostate cancer is the most common cancer in men, accounting for $15 \%$ of all diagnosed cancers and being the sixth leading cause of cancer-related deaths amongst men worldwide.

Metastatic prostatic cancer can be roughly divided in two main clinical stages: hormone-sensitive and castrationresistant prostate cancer.
Since 1941, when Charles Huggins and colleagues uncovered the hormonal dependence of metastatic prostate cancer, androgen deprivation therapy has been the therapeutical mainstay for metastatic prostate cancer.

After decades of absent novelties, we assisted to the introduction of novel hormonal agents for the treatment of castration-resistant metastatic prostate cancer.

Abiraterone and enzalutamide were the first two novel hormonal agents approved for the treatment of metastatic prostate cancer and are now widely used. Abiraterone is an inhibitor of CYP17A1, an enzyme essential in the process of androgen synthesis. Enzalutamide competitively inhibits androgen binding to the androgen receptor (AR), nuclear translocation of the AR, DNA binding and coactivator recruitment. Both abiraterone and enzalutamide are first line treatments for metastatic castrationresistant prostate cancer ( $\mathrm{MCRPC}$ ), but there is a lack of quality evidence regarding which is associated with better outcomes and who would benefit the most with one or another of these drugs.

Our propose was to evaluate the clinical outcomes of patients submitted to either abiraterone or enzalutamide for castration-resistant metastatic prostate cancer in our center.

\section{PATIENTS AND METHOdS}

Patients eligible for this study had a diagnosis of metastatic castration-resistant prostate cancer defined as castrate serum testosterone $<50 \mathrm{ng} / \mathrm{mL}$ and biochemical progression (three consecutives rises in prostatic specific antigen (PSA) at least one week apart resulting in two 50\% increases over the nadir, and a PSA $>2 \mathrm{ng} / \mathrm{mL}$ ) or radiological progression (appearance of new lesions: either two or more new bone lesions or a soft lesion using Response Evaluation Criteria in Solid Tumours (RECIST)) and initiated treatment with either abiraterone or enzalutamide between January 1 , 2016 and December 31, 2019. Follow-up extended from January 1, 2016 until December 31, 2020. In 2019, we treated 358 patients diagnosed with prostate cancer, representing roughly $5.5 \%$ cases nationwide.

Individual clinical cases were discussed in bi-weekly oncourology meetings. Patients with clinical criteria of poor prognosis (symptoms, short period of response under

No conflict of interest declared. 
androgen deprivation therapy (ADT), high metastatic burden, visceral metastasis or poor prognostic genetic mutations) were treated with taxane-based chemotherapy. The decision to begin treatment with a novel hormonal agent was taken either as first line therapy in patients with less aggressive features (asymptomatic, durable response under previous ADT, low metastatic burden and no visceral metastases) or as second line therapy in patients that progressed under first line therapy with taxane-based chemoterapy. In the absence of contraindication for either pharmaceutical drugs, patients were sequentially assigned to either enzalutamide or abiraterone group.

The database used was anonymized and unstructured. Data were originally extracted from electronic medical records. Demographic and clinicopathological features (International Society of Urological Pathology (ISUP) score, Ml $a b$ inition or progression after local treatment status, previous period of classical androgen deprivation therapy, PSA level, previous treatment of taxane-based chemotherapy and localization of metastasis) were registered at baseline. Data reported adverse events (AEs) was also available.

Per local protocol, stable patients with metastatic prostate cancer under novel hormonal agents are followed with clinical and analytical evaluation including PSA measurement every 3 months by a staff expert. Occurrence of biochemical response (defined as a reduction of $\geq 50 \%$ of pretreatment PSA level after 12 weeks of treatment), PSA progression (three consecutives rises in PSA at least one week apart resulting in two 50\% increases over the nadir, and a PSA $>2 \mathrm{ng} / \mathrm{mL}$ ), radiological progression (appearance of new lesions: either two or more new bone lesions or a soft lesion using RECIST) diagnosed either by computed tomography (CT) scan, bone scintigraphy or G68 prostate-specific membrane antigen (PSMA) - positron emission tomography (PET), clinical deterioration and/or death and the time of their occurrence were available.

The primary endpoints of this study were biochemical response, biochemical progression, radiological progression, clinical deterioration (attributed to disease progression) and death. Safety was a secondary endpoint.

Follow-up was stopped when the drug was suspended due to adverse reactions or disease progression. There was no crossover.

\section{Statistical analysis}

Descriptive statistics of patient and pathological characteristic were calculated for all patients included in the present study, as well as by administered agent.

We used a chi-square univariate analysis to assess the statistical significance of the difference between rate response in the abiraterone and enzalutamide group. Further, we used a binary logistic regression, adjusted to clinicopathological features, to quantify this association. A Cox-regression was performed to uncover predictive co-variates of biochemical progression, radiological progression and overall survival.

A survival analysis using a Kaplan-Meier method was used to evaluate the risk of biochemical progression, radiological progression and overall survival and a log-rank test applied to test for significant differences.

All analyses were conducted using IBM SPSS statistics version 23. All comparisons were made using 2-sided tests, with $\mathrm{p}<0.05$ considered statistically significant.

\section{Results}

A total of 91 patients who initiated a novel hormonal agent, either abiraterone or enzalutamide, between January 2016 and December 2019 were included in the study. $56(61.5 \%)$ patients were treated with abiraterone and the remaining 35 (38.5\%) with enzalutamide. 45 (49.5\%) patients were diagnosed with metastatic prostate cancer ab inition. $74.7 \%, 53.8 \%$ and $6.6 \%$ had bone, lymphatic, and visceral metastasis, respectively. Almost 30\% of patients had been previously submitted to taxane-based chemotherapy. Mean age of the cohort was $74.4( \pm 8.26)$ years, while the mean pretreatment PSA level was 231.51 $( \pm 380.15) \mathrm{ng} / \mathrm{mL}$ and previous classical androgen deprivation therapy duration was $74.13( \pm 54.87)$ months. Median follow-up time was 18.34 months.

All covariates, as age, pretreatment PSA level, ISUP score, Ml ab inition or progression after local treatment status, previous period of classical androgen deprivation therapy, previous treatment of taxane-based chemotherapy and localization of metastasis were similar between the groups. Baseline demographic and clinicopathological features are shown in Table 1.

\section{Biochemical response}

Overall, rate of biochemical response was $61.5 \%$ in mCRPC patients. It was found to be significantly higher in the enzalutamide group than in the abiraterone group, with a rate response of $77.1 \%$ and $58.1 \%$, respectively $(\mathrm{p}=0.016)$. In binary logistic regression adjusted to clinicopathological features, enzalutamide was associated with a higher proba-

\section{Table 1.}

Baseline demographic and clinicopathological characteristics by drug of mCRPC patients.

\begin{tabular}{|c|c|c|c|c|}
\hline \multicolumn{5}{|c|}{ Demographic and pathological features } \\
\hline & $\begin{array}{c}\text { Total } \\
(n=91)\end{array}$ & $\begin{array}{l}\text { Abiraterone } \\
(\mathrm{n}=56)\end{array}$ & $\begin{array}{c}\text { Enzalutamide } \\
(\mathrm{n}=35)\end{array}$ & $\mathbf{P}$ \\
\hline Age (years) & $74.44 \pm 8.26$ & $75.00 \pm 7.01$ & $73.54 \pm 9.980$ & 0.416 \\
\hline Pretreatment PSA level (ng/ml) & $231.51 \pm 380.16$ & $215.40 \pm 372.82$ & $257.94 \pm 401.65$ & 0.717 \\
\hline$\overline{\text { Previous ADT (months) }}$ & $74.13 \pm 54.87$ & $77.53 \pm 61.66$ & $68.82 \pm 42.50$ & 0.882 \\
\hline Follow-up (months) & $18.34 \pm 9.81$ & $17.16 \pm 8.71$ & $20.68 \pm 11.20$ & 0.276 \\
\hline$\overline{\text { ISUP }}$ & & & & 0.612 \\
\hline 1 & $9(10 \%)$ & $4(7.3 \%)$ & $5(14.3 \%)$ & \\
\hline 2 & $18(20 \%)$ & $13(23.6 \%)$ & $5(14.3 \%)$ & \\
\hline 3 & $25(27.8 \%)$ & $16(29.1 \%)$ & $9(25.7 \%)$ & \\
\hline$\geq 4$ & $19(21.1 \%)$ & $12(21.8 \%)$ & $7(20 \%)$ & \\
\hline$N / A$ & $19(21.1 \%)$ & $10(18.2 \%)$ & $9(25.7 \%)$ & \\
\hline Status & & & & 0.934 \\
\hline M1 ab inition & $45(50.6 \%)$ & $28(50.9 \%)$ & $17(50 \%)$ & \\
\hline Post local treatment & $44(49.4 \%)$ & $27(49.1 \%)$ & $17(50 \%)$ & \\
\hline$\overline{\text { Metastasis }}$ & & & & \\
\hline Bone & $68(74.7 \%)$ & $39(69.6 \%)$ & $29(82.9 \%)$ & 0.158 \\
\hline Ganglionar & $49(53.8 \%)$ & $30(53.6 \%)$ & $19(54.3 \%)$ & 0.947 \\
\hline Visceral & $6(6.2 \%)$ & $4(6.5 \%)$ & $2(5.7 \%)$ & 0.788 \\
\hline Post docetaxel & $27(29.7 \%)$ & $15(26.8 \%)$ & $12(34.3 \%)$ & 0.446 \\
\hline
\end{tabular}




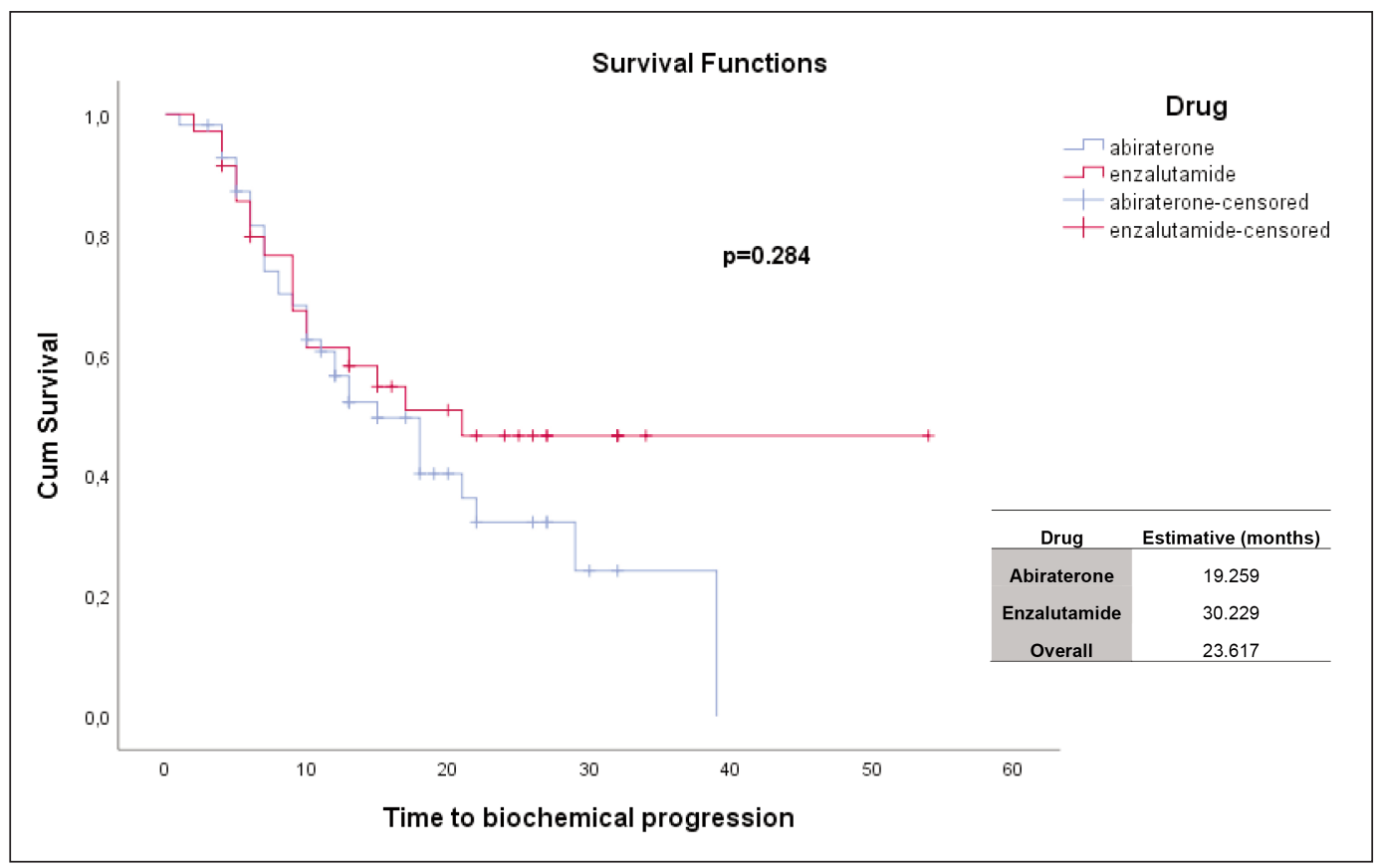

Figure 1.

Biochemical progression based on administered

drug.

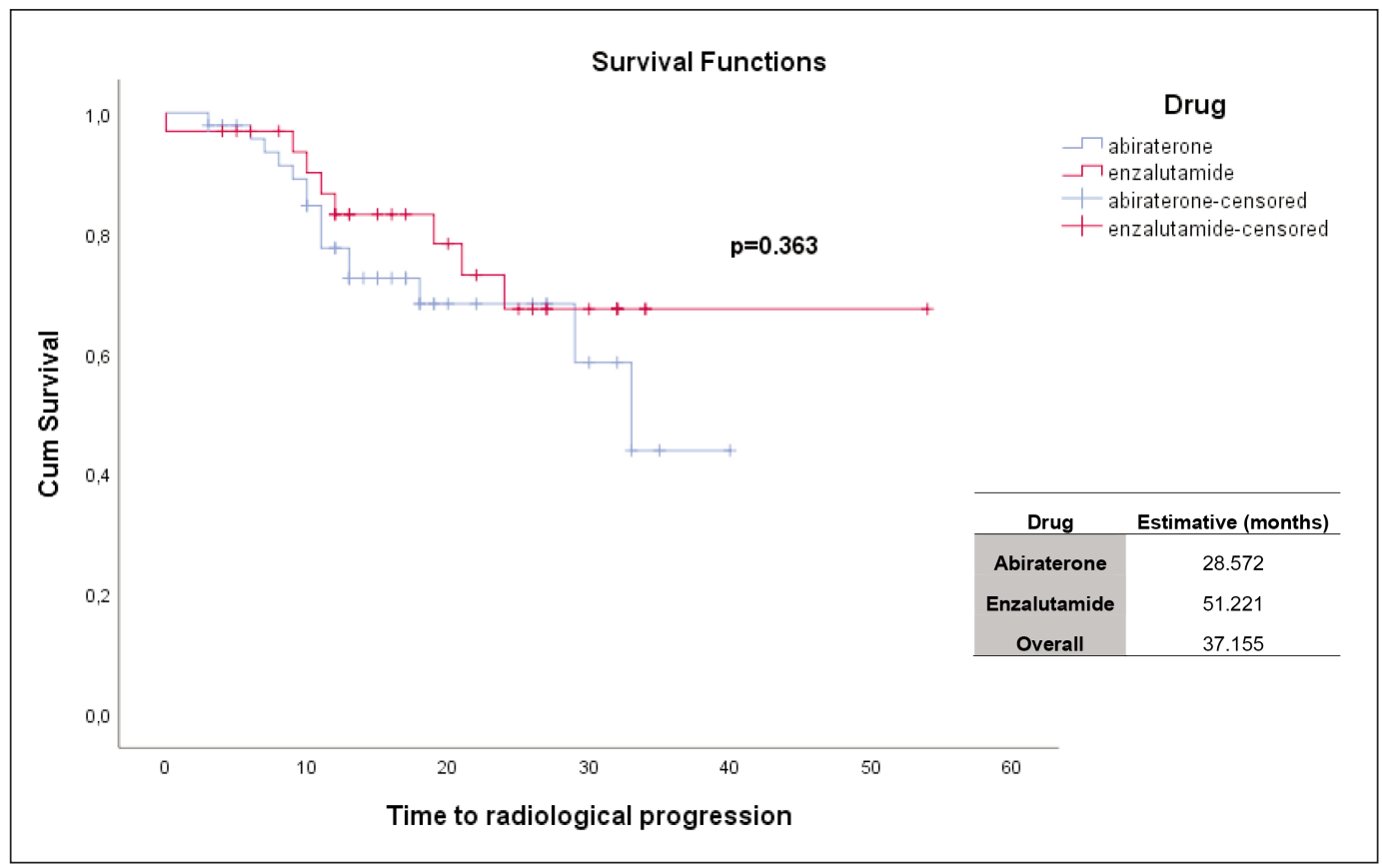

Figure 2.

Radiological progression based on administered drug. bility of biochemical response (OR: 3.485, $\mathrm{p}=0.021$ ). Biochemical response was associated with lower probability of biochemical progression (OR: $0.248, \mathrm{p}=0.017$ ) and death (OR: 0.302, $\mathrm{p}=0.038$ ), adjusted to clinicopathological features.

Subgroup analyses showed no statistically significant difference between biochemical responses in the docetaxelnaïve patients between abiraterone and enzalutamide (56.1\% vs $73.9 \%, p=0.158$ ), but enzalutamide had a higher response rate than abiraterone in patients previously submitted to docetaxel ( $83.3 \%$ vs 40\%, p = 0.019). In the subgroup of patients with metastatic prostate cancer $a b$ inition, that progressed to $\mathrm{MCRPC}$ and were docetaxel-naive, the period of previous classical hormonal therapy was inversely associated with biochemical response (OR: 0.928, $\mathrm{p}=0.035$ ).

\section{Biochemical progression}

mCRPC patients submitted to treatment with enzalutamide and abiraterone had a biochemical progressionfree survival (bPFS) of 19.2 and 30.2 months, respectively. The difference failed to achieve statistical significance $(\mathrm{p}=0.284)$. The Kaplan-Meier curves for bPFS are showed in the Figure 1. In patients who achieved a biochemical response, the bPFS was similar in both enzalutamide group and abiraterone group (24.0 vs 24.3 months, $p=0.651$ ). No covariate factor was identified as predictor of bPFS in multivariate analysis. 


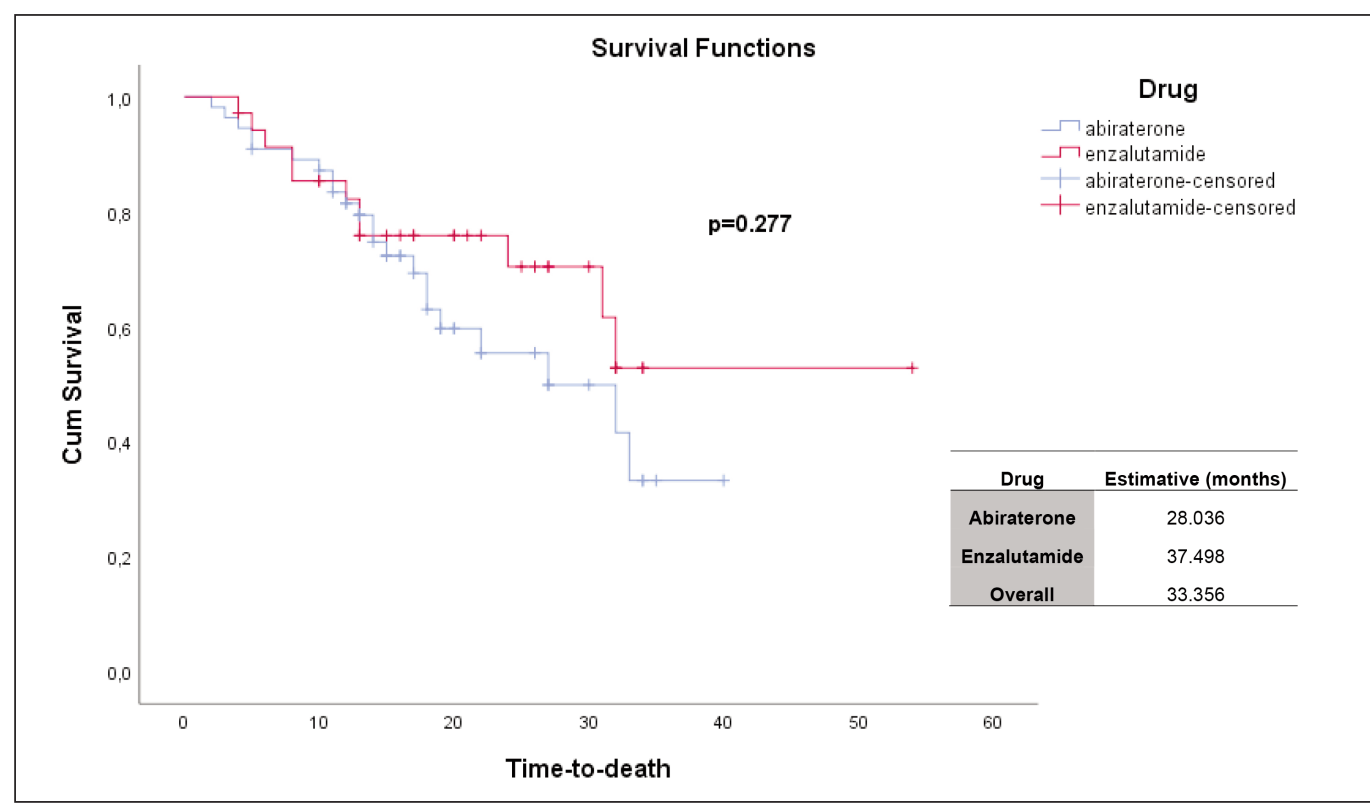

Figure 3.

Overall survival based on administered drug.

\section{Radiological progression}

Overall, in the mCRPC group the rPFS in patients submitted to enzalutamide was 41.2 months vs 28.57 months in the abiraterone group, but with no statistical significance $(p=0.363)$. The Kaplan-Meier curves for rPFS are showed in the Figure 2. Among biochemical responders, patients submitted to abiraterone had a rPFS of 32.2 months and patients treated with enzalutamide had a rPFS of 29.34 months. No statistically significant differences was achieved $(p=0.791)$.

No covariate factor was identified as predictor of rPFS in multivariate analysis.

\section{Overall survival}

All cause time-to-death, in mCRPC patients, was 37.5 months in enzalutamide group and 26 months in abiraterone group, without achieving a statistically significant difference $(p=0.277)$. The Kaplan-Meier curves for overall survival (OS) are showed in the Figure 3.

In patients in whom biochemical response was achieved, the abiraterone group had a OS of 31.27 months vs 27.30 months in the enzalutamide group, but without statistically significant difference $(\mathrm{p}=0.994)$.

In patients that failed to meet biochemical response criteria, OS was 31.15 months and 17.58 months in enzalutamide and abiraterone group, respectively. A statistically significant difference was not achieved (31.18 vs 17.58, $\mathrm{p}=0.121$ ). No covariate factor was identified as predictor of OS in multivariate analysis.

\section{AEs associated with treatment}

\section{with abiraterone or enzalutamide}

Overall, 14 patients (14.4\%) experienced drug-related adverse events (AEs), 10 (16.1\%) in abiraterone group and $4(11.4 \%)$ in enzalutamide group. The common AEs that occurred in this series were as follows: fatigue $(60 \%)$ and diarrhea (20\%) in abiraterone group and fatigue (100\%) in enzalutamide group.

Only 2 AEs $\geq$ grade 3 were registered, 1 in each group, causing the suspension of the drug.

\section{Discussion}

Novel hormonal agents are now a cornerstone in the treatment of castration-resistant prostate cancer. Both enzalutamide and abiraterone with prednisolone are approved therapies for men with mCRPC. These two drugs have shown clinical efficacy in multicenter phase III RCTs (1-4), yet there is a lack of evidence regarding comparative outcomes of men submitted to treatment with either of the two drugs. Findings from previous retrospective studies suggest survival advantages toward enzalutamide compared with abiraterone and prednisolone in the treatment of men with mCRPC (5), although both drugs were effective, with PSA rate response over $50 \%$. Our analysis is consistent with prior data for better biochemical response with enzalutamide in men with mCRPC. We found that patients in the enzalutamide group had a significantly higher biochemical response than the abiraterone group, with a rate response of $51.8 \%$ and $77.1 \%$, respectively $(\mathrm{p}=0.016)$. This seems particularly relevant in patients previously submitted to taxane-based chemotherapy. In a multivariate analysis, enzalutamide was associated with a higher probability of rate response compared with abiraterone (OR: 3.485, $\mathrm{p}=0.021$ ). Biochemical response was associated with a lower probability of biochemical progression (OR: 0.248, $\mathrm{p}=0.017)$ and death (OR: 0.302, $\mathrm{p}=0.038)$. This data suggests that the higher rate of biochemical response of enzalutamide is associated with better outcomes.

Respective to other endpoints (biochemical progression, radiological progression, and overall survival), there was a trend toward advantage of enzalutamide over abiraterone with no statistical significance. Data showed that in patients who achieved biochemical response, the apparent advantage of enzalutamide over abiraterone disappears. Patients who did not achieve a biochemical response had a trend to longer survival when treated to enzalutamide vs abiraterone, albeit not statistically different. As suggested in previous studies $(6,7)$, this may support an early change of therapeutical strategy when biochemical response is not achieved with abiraterone. 
Miyake et al., retrospectively reviewed 280 docetaxelnaïve $\mathrm{mCRPC}$ patients. A higher PSA response rate for patients treated with enzalutamide when compared with abiraterone (70.7\% vs 53.1\%) was found, in line with our results. On the other hand, a better bPFS in enzalutamide group was also found, which our study failed to find. In this study, because of the drug choice was at physician discretion, there was a preference of abiraterone over enzalutamide in patients with less favorable clinicopathological features based in the results of COU-AA-302 and PREVAIL trials, respectively (8).

Heo et al. performed a retrospective study that evaluated the outcomes of patients diagnosed with mCRPC treated with abiraterone and enzalutamide in post-docetaxel setting. 54 patients were evaluated ( 25 in abiraterone group and 29 in enzalutamide group) and a PSA rate response was seen $36 \%$ and $52 \%$ for abiraterone and enzalutamide, respectively. In our cohort the PSA rate response was similar for abiraterone (40\%), but enzalutamide had a higher response rate than reported in this study (83.3\%) in this subset of patients (9).

Both drugs were well tolerated, with low incidence of drug-related grade $\geq 3$ events. Only two patients had their treatment suspended. The type of AEs registered were in line with ones reported in COU-AA-302 and PREVAIL trials.

Norris et al. retrospectively studied 198 mCRPC patients submitted to treatment with abiraterone and enzalutamide. Significantly higher PSA response rates were observed in the enzalutamide (51\%) than abiraterone (36\%). In our cohort the overall PSA response rate were higher, $51.8 \%$ and $77.1 \%$ in abiraterone and enzalutamide, respectively. There was no significant difference in OS between the groups with median OS of 15.3 months in abiraterone group versus 22.2 months in the enzalutamide group. The OS survival in our study was also higher for enzalutamide (37.5 months for enzalutamide and 27.30 months for abiraterone). These differences can be partially explained by the proportion of patients that were treated in post-docetaxel setting in each study, being the majority of patients in Norris et al. cohort and only $30 \%$ in ours. As seen in our cohort, higher PSA response rates were seen in the pre-docetaxel group compared to the post-docetaxel (10).

Garcia et al. performed a retrospective observational study reviewing 48 patients with MCRPC (26 in abiraterone group and 22 in enzalutamide). Most patients had been submitted to docetaxel. The primary endpoint was biochemical response. Unlike our study, no statistically difference in biochemical response between abiraterone and enzalutamide was observed $(53.85 \%$ and $58.85 \%$ for abiraterone and enzalutamide groups, respectively). A low number of patients treated can impair the statistical power of this study (11).

Khalaf et al. retrospectively analyzed 210 patients (106 in abiraterone and 104 in enzalutamide groups), older than 80 years who received novel hormonal agents for firstline treatment of mCRPC. As in our cohort, the biochemical response was higher in patients treated with enzalutamide than abiraterone (77.9\% vs $43.4 \%$ ) but no OS advantage was observed. In our study the PSA response rates of docetaxel-naive patients were similar with the ones reported in this study (56.1\% and $73.9 \%$ for abiraterone and enzalutamide, respectively) (12).

Our study has some strengths: similar groups regarding clinicopathological characteristics and a relatively uniform distribution between abiraterone and enzalutamide groups ( $61.5 \%$ vs $38.5 \%)$. A $1: 1$ proportion between abiraterone and enzalutamide was not achieved due to the numerous contraindications to enzalutamide.

The approval of abiraterone for treatment of MCRPC was conceded before enzalutamide by national regulatory agency, contributing to the discrepancy in the number of patients in each group.

Some limitations of this study must however be noted. As a real-life study, the results can be biased by not randomized allocation of patients to different treatments. Adverse events were not systematically evaluated due to retrospective nature of this study, making security performance assessment inaccurate. The threshold and image modality (either CT and bone scintigraphy or Ga68 PETPSMA) used for staging patients in the scenario of biochemical progression was not specified, introducing some bias in radiological progression assessment. Mean followup period was relatively short.

Based on our results, enzalutamide conferred higher biochemical response rate than abiraterone in patients with mCRPC. Achieving a biochemical response was associated with a lower risk of biochemical progression and death. Other endpoints tended to improve in the enzalutamide group although not significantly. Well designed prospective studies are needed to elucidate the comparative efficacies of these agents.

\section{REFERENCES}

1. Fizazi K, Scher HI, Molina A, et al. Abiraterone acetate for treatment of metastatic castration-resistant prostate cancer: final overall survival analysis of the COU-AA-301 randomised, double-blind, placebo-controlled phase 3 study. Lancet Oncol. 2012; 13:983-92.

2. Beer TM, Armstrong AJ, Rathkopf DE, et al. Enzalutamide in metastatic prostate cancer before chemotherapy. N Engl J Med. 2014; 371:424-33.

3. Scher HI, Fizazi K, Saad F, et al. Increased survival with enzalutamide in prostate cancer after chemotherapy. N Engl J Med. 2012; 367:1187-97.

4. Ryan CJ, Smith MR, Fizazi K, et al. Abiraterone acetate plus prednisone versus placebo plus prednisone in chemotherapy-naive men with metastatic castration-resistant prostate cancer (COU-AA-302): final overall survival analysis of a randomised, double-blind, placebo-controlled phase 3 study. Lancet Oncol. 2015; 16:152-60.

5. Wang X, Yang H, Hu X, et al. Comparing the clinical efficacy and safety of abiraterone and enzalutamide in metastatic castrationresistant prostate cancer: A systematic review and meta-analysis. J Oncol Pharm Pract. 2021; 27:614-622.

6. Khalaf DJ, Annala M, Taavitsainen S, et al. Optimal sequencing of enzalutamide and abiraterone acetate plus prednisone in metastatic castration-resistant prostate cancer: a multicentre, randomised, open-label, phase 2, crossover trial. Lancet Oncol. 2019; 20:1730-9.

7. Hung SC, Wang SS, Li JR, et al. Outcome of Patients with Metastatic Castration-resistant Prostate Cancer After PSA Progression with Abiraterone Acetate. Anticancer Res. 2018; 38:5429-36. 
8. Miyake H, Hara T, Terakawa T, et al. Comparative assessment of clinical outcomes between abiraterone acetate and enzalutamide in patients with docetaxel-naive metastatic castration-resistant prostate cancer: experience in real-world clinical practice in Japan. Clin Genitourin Cancer. 2017; 15:313-9.

9. Heo MH, Park SH, Kim HK, et al. Overall survival beyond firstline docetaxel in patients with metastatic castrate-resistant prostate cancer treated with abiraterone acetate or enzalutamide. J Clin Oncol. 2017; 35(6_suppl):e570-e570.

10. Norris T, Walter S, Williams A, et al. Comparison of Toxicity and Efficacy Outcomes of Abiraterone and Enzalutamide in 198
Patients with Metastatic Castrate Resistant Prostate Cancer. Clin Oncol. 2017; 29:e87-8.

11. García AS, Mateos AA, Esquerdo ML, et al. 4CPS-128 Effectiveness of abiraterone acetate and enzalutamide in metastatic castration-resistant prostate cancer. Eur J Hosp Pharm. 2018; 25(Suppl 1):A101-A101.

12. Khalaf D, Zou K, Struss WJ, et al. Efficacy and tolerability of first-line abiraterone + prednisone (ABI) versus enzalutamide (ENZ) for metastatic castration-resistant prostate cancer ( $m C R P C)$ in men $\geq 80$ years: A retrospective cohort study. J Clin Oncol. 2018; 36(15_suppl):5051-5051.

\section{Correspondence}

Roberto Saldanha Jarimba

robertojarimba@chuc.min-saude.pt

Serviço de Urologia, Centro Hospitalar e Universitário de Coimbra Rua Professor Mota Pinto 3004-561, Coimbra (Portugal)

Miguel Nobre Eliseu, MD

João Pedroso Lima, MD

Vasco Quaresma, MD

Pedro Moreira, MD

Urology and Renal Transplantation Department, Centro Hospitalar e Universitário de Coimbra, Coimbra (Portugal)

Pedro Coelho Nunes, MD

Edgar Tavares da Silva, MD

Arnaldo José Figueiredo, MD

Urology and Renal Transplantation Department, Centro Hospitalar

e Universitário de Coimbra \& Faculty of Medicine, University of Coimbra,

Coimbra (Portugal) 